\title{
Polar subspaces and automatic maximality
}

\author{
S. Simons \\ Department of Mathematics, University of California \\ Santa Barbara, CA 93106-3080, U.S.A. \\ simons@math.ucsb.edu
}

\begin{abstract}
This paper is about certain linear subspaces of Banach SN spaces (that is to say Banach spaces which have a symmetric nonexpansive linear map into their dual spaces). We apply our results to monotone linear subspaces of the product of a Banach space and its dual. In this paper, we establish several new results and also give improved proofs of some known ones in both the general and the special contexts.
\end{abstract}

\section{Introduction}

This paper is a sequel to [14], in which we developed the theory of Banach SN spaces (that is to say Banach spaces which have a symmetric nonexpansive linear map into their dual spaces) and " $L$-positive" sets, and applied it to a number of results about monotone linear subspaces of the product of a Banach space and its dual. Banach SN spaces, L-positive sets and Banach SN duals are defined formally in Sections 3 and 4.

Section 5 is about closed linear $L$-positive subspaces of those Banach SN spaces that possess Banach SN duals. The basic technical result of this paper is Lemma 5.5. The novelty of our proof is that we use a penalty function of the form $2 \eta\|\cdot\|$ rather than $\frac{1}{2}\|\cdot\|^{2}$. This not only produces significantly sharper results, but also leads to simpler proofs than those previously given for results of this kind. We actually use two special cases of Lemma 5.5: Corollary 5.6 and Corollary 5.7.

The most important consequences of Lemma 5.5 are Theorem 5.9 and Corollary 5.11. In Remarks 5.8 and 5.10, we give details of the connections between these results and those already proved in [14].

The results on monotone subspaces in Theorem 6.4, Corollary 6.6 and Corollary 6.7 all have one-line proofs using the corresponding results on Banach SN spaces, and these latter results have much simpler proofs that those that we have seen in the literature in the monotone case. In Theorem 6.4 we establish the following result on "automatic maximality", which we have not seen in the literature: Let $A$ be a closed linear monotone subspace of product of a real Banach space with its dual, and the adjoint subspace of $A$ be monotone. Then $A$ and its adjoint are both maximally monotone in their respective spaces. Theorem 6.4 and Corollary 6.6 are discussed in Remark 6.5.

The motivating result behind this analysis is the classical Brezis-Browder theorem (see [2]): Let $A$ be a closed linear monotone subspace of the product of a reflexive real Banach space with its dual space. Then the adjoint subspace of $A$ is monotone if, and only if, $A$ is maximally monotone. This was generalized by Bauschke, Borwein, Wang and Yao (see [3] and [5]): If $A$ is a closed linear monotone subspace of the product of a real Banach space with its dual space then the adjoint subspace of $A$ is monotone if, and only if, $A$ is maximally monotone of type (NI). See Corollary 6.6 for our generalization of this and Corollary 6.7 for a proof of the original Brezis-Browder theorem. 


\section{Polar subspaces and automatic maximality}

We use some basic tools of convex analysis (that is to say Rockafellar's formula for the subdifferential of a sum, and the Brøndsted-Rockafellar theorem). In addition, we use two simple preliminary results, Lemma 2.2 and Lemma 3.3.

The author would like to thank Liangjin Yao for sending him the preprints [3], [4], [5] and [15], and Maicon Marques Alves for sending him the preprint [9].

\section{Banach space notation - Polar subspaces}

We start off by introducing some Banach space notation.

Definition 2.1. If $X$ is a nonzero real Banach space and $f: X \rightarrow]-\infty, \infty]$, we say that $f$ is proper if there exists $x \in X$ such that $f(x) \in \mathbb{R}$. We write $X^{*}$ for the dual space of $X$ (with the pairing $\langle\cdot, \cdot\rangle: X \times X^{*} \rightarrow \mathbb{R}$ ) and $X^{* *}$ for the bidual of $X$ (with the pairing $\left.\langle\cdot, \cdot\rangle: X^{*} \times X^{* *} \rightarrow \mathbb{R}\right)$. If $x \in X$, we write $\widehat{x}$ for the canonical image of $x$ in $X^{* *}$, that is to say $x \in X$ and $x^{*} \in X^{*} \Longrightarrow\left\langle x^{*}, \widehat{x}\right\rangle=\left\langle x, x^{*}\right\rangle$. We write "lin" for "linar span of". If $Y$ is a linear subspace of $X$, we write $Y^{0}$ for the "polar subspace of $Y$ ", that is to say the linear subspace $\left\{x^{*} \in X^{*}:\left\langle Y, x^{*}\right\rangle=\{0\}\right\}$ of $X^{*}$.

A word is in order about the proof of Lemma 2.2 below. The proof we give is an adaptation of a classical proof of a result about linear functionals (see, for instance, [8, Theorem 1.3, p, 7]). However, it is worth pointing out that when $Y$ is closed the result also follows from the Robinson-Attouch-Brezis theorem applied to the indicator functions of $Y$ and $Z$. We will use Lemma 2.2 in Theorem 5.9(b).

Lemma 2.2. Let $Y$ be a linear subspace of a real Banach space $X$ and $x^{*} \in X^{*}$. Write $Z$ for the linear subspace $\left\{x \in X:\left\langle x, x^{*}\right\rangle=0\right\}$ of $X$. Then $(Y \cap Z)^{0}=\operatorname{lin}\left\{x^{*}, Y^{0}\right\}$.

Proof. Since $x^{*} \in Z^{0} \subset(Y \cap Z)^{0}$ and $Y^{0} \subset(Y \cap Z)^{0}$, and $(Y \cap Z)^{0}$ is a linear subspace, it is immediate that $\operatorname{lin}\left\{x^{*}, Y^{0}\right\} \subset(Y \cap Z)^{0}$.

We now prove the opposite inclusion. If $Y \subset Z$ then $Y \cap Z=Y$, so $(Y \cap Z)^{0}=Y^{0} \subset$ $\operatorname{lin}\left\{x^{*}, Y^{0}\right\}$, as required. We now come to the more interesting case, where $Y \not \subset Z$. We fix $x \in Y \backslash Z$, from which $\left\langle x, x^{*}\right\rangle \neq 0$. Let $y$ be an arbitrary element of $Y$, and define

$$
z:=y-\frac{\left\langle y, x^{*}\right\rangle}{\left\langle x, x^{*}\right\rangle} x \in X
$$

Since $y, x \in Y$, it follows that $z \in Y$. Since

$$
\left\langle z, x^{*}\right\rangle=\left\langle y, x^{*}\right\rangle-\frac{\left\langle y, x^{*}\right\rangle}{\left\langle x, x^{*}\right\rangle}\left\langle x, x^{*}\right\rangle=0,
$$

we have $z \in Z$. Thus $z \in Y \cap Z$. Now let $z^{*}$ be an arbitrary element of $(Y \cap Z)^{0}$. Then

$$
0=\left\langle z, z^{*}\right\rangle=\left\langle y, z^{*}\right\rangle-\frac{\left\langle y, x^{*}\right\rangle}{\left\langle x, x^{*}\right\rangle}\left\langle x, z^{*}\right\rangle=\left\langle y, z^{*}-\frac{\left\langle x, z^{*}\right\rangle}{\left\langle x, x^{*}\right\rangle} x^{*}\right\rangle .
$$

Since this holds for all $y \in Y$,

$$
z^{*}-\frac{\left\langle x, z^{*}\right\rangle}{\left\langle x, x^{*}\right\rangle} x^{*} \in Y^{0} .
$$

But then

$$
z^{*} \in \frac{\left\langle x, z^{*}\right\rangle}{\left\langle x, x^{*}\right\rangle} x^{*}+Y^{0} \in \operatorname{lin}\left\{x^{*}, Y^{0}\right\} .
$$

This completes the proof of Lemma 2.2. 


\section{Polar subspaces and automatic maximality}

\section{Banach SN spaces}

We now introduce Banach SN spaces and $L$-positive sets ([14, Section 2, pp. 604-606]). (These were called Banach SNL spaces in [14].)

Definition 3.1. Let $B$ be a nonzero real Banach space. A $S N$ map on $B$ ("SN" stands for "symmetric nonexpansive"), is a linear map $L: B \rightarrow B^{*}$ such that

$$
\|L\| \leq 1 \quad \text { and }, \quad \text { for all } b, c \in B, \quad\langle b, L c\rangle=\langle c, L b\rangle .
$$

A Banach $S N$ space $(B, L)$ is a nonzero real Banach space $B$ together with a SN map $L: B \rightarrow B^{*}$. We define the function $q_{L}: B \rightarrow \mathbb{R}$ by $q_{L}(b):=\frac{1}{2}\langle b, L b\rangle \quad(b \in B) \quad$ (" $q$ " stands for "quadratic"). It follows from (1) that, for all $a, b \in B$,

$$
\left|q_{L}(a)-q_{L}(b)\right|=\frac{1}{2}|\langle a, L a\rangle-\langle b, L b\rangle|=\frac{1}{2}|\langle a-b, L(a+b)\rangle| \leq \frac{1}{2}\|a-b\|\|a+b\|,
$$

and so

$$
q_{L} \text { is continuous on } B \text {. }
$$

Now let $(B, L)$ be a Banach SN space and $A \subset B$. We say that $A$ is $L$-positive if $A \neq \emptyset$ and $\quad b, c \in A \Longrightarrow q_{L}(b-c) \geq 0$. We say that $A$ is maximally $L$-positive if $A$ is $L$-positive and $A$ is not properly contained in any other $L$-positive set. In this case,

$$
b \in B \Longrightarrow \inf _{a \in A} q_{L}(a-b) \leq 0 .
$$

(If $d \in B \backslash A$ then the maximality gives us $a \in A$ such that $q_{L}(a-d)<0$, while if $d \in A$ then $q_{L}(d-d)=0$.)

There are many examples of Banach SN spaces and their associated $L$-positive sets. The following are derived from [13, Examples 2.3, 2.5, pp. 230-231]. More examples can be derived from [13, Remark 6.7, p. 246] and [7]. The significant example which leads to results on monotonicity appeared in [13, Example 6.5, p. 245]. We will return to it here in Example 6.1.

Example 3.2. Let $B$ be a Hilbert space with inner product $(b, c) \mapsto\langle b, c\rangle$ and $L: B \rightarrow B$ be a nonexpansive self-adjoint linear operator. Then $(B, L)$ is a Banach SN space. Here are three special cases of this example:

(a) If, for all $b \in B, L b=b$ then every subset of $B$ is $L$-positive.

(b) If, for all $b \in B, L b=-b$ then the $L$-positive sets are the singletons.

(c) If $B=\mathbb{R}^{3}$ and $L\left(b_{1}, b_{2}, b_{3}\right)=\left(b_{2}, b_{1}, b_{3}\right)$ and $M$ is any nonempty monotone subset of $\mathbb{R} \times \mathbb{R}$ (in the obvious sense) then $M \times \mathbb{R}$ is a $L$-positive subset of $B$. The set $\mathbb{R}(1,-1,2)$ is a $L$-positive subset of $B$ which is not contained in a set $M \times \mathbb{R}$ for any monotone subset of $\mathbb{R} \times \mathbb{R}$. The helix $\{(\cos \theta, \sin \theta, \theta): \theta \in \mathbb{R}\}$ is a $L$-positive subset of $B$, but if $0<\lambda<1$ then the helix $\{(\cos \theta, \sin \theta, \lambda \theta): \theta \in \mathbb{R}\}$ is not.

(d) If $B=\mathbb{R}^{3}$ and $L\left(b_{1}, b_{2}, b_{3}\right)=\left(b_{2}, b_{3}, b_{1}\right)$ then, since (1) fails, $(B, L)$ cannot be a Banach SN space.

We will use the following special property of $L$-positive linear subspaces in Theorem 5.9(b): 


\section{Polar subspaces and automatic maximality}

Lemma 3.3. Let $A$ be a linear subspace of $B, b \in B$ and $\{b\} \cup A$ be $L$-positive. Then the linear subspace $\operatorname{lin}\{b, A\}$ of $B$ is $L$-positive.

Proof. Let $C:=\operatorname{lin}\{b, A\}$ and $c \in C$. Then there exist $\lambda \in \mathbb{R}$ and $a \in A$ such that $c=\lambda b-a$. If $\lambda=0$ then, since $0, a \in A \subset\{b\} \cup A$ and $\{b\} \cup A$ is $L$-positive, $q_{L}(c)=q_{L}(0-a) \geq 0$. If $\lambda \neq 0$ then, since $b \in\{b\} \cup A, \lambda^{-1} a \in A \subset\{b\} \cup A$ and $\{b\} \cup A$ is $L$-positive, $q_{L}(c)=\lambda^{2} q_{L}\left(b-\lambda^{-1} a\right) \geq 0$. Thus $q_{L} \geq 0$ on $C$. Since $C$ is a linear subspace of $B, C$ is $L$-positive.

\section{Banach SN duals}

Definition 4.1. Let $(B, L)$ be a Banach $\mathrm{SN}$ space and $\left(B^{*}, \widetilde{L}\right)$ also be a Banach $\mathrm{SN}$ space. We say that $\left(B^{*}, \widetilde{L}\right)$ is a Banach $S N$ dual of $(B, L)([14$, Section 2, pp. 604-606]) if

$$
b \in B \text { and } b^{*} \in B^{*} \quad \Longrightarrow\left\langle L b, \widetilde{L} b^{*}\right\rangle=\left\langle b, b^{*}\right\rangle .
$$

This is equivalent to the definition introduced in [14, Eq. (5), p. 605].

In the examples introduced in Example 3.2(a,b,c), the linear map $L$ is orthogonal, from which $(B, L)$ is a Banach SN dual of itself. However, not all Banach SN spaces have Banach SN duals - it was shown in [14, Lemma 8.1, p. 614] that if $(B, L)$ is a Banach SN space with a Banach SN dual then $L$ is necessarily an isometry, consequently if $B$ is nonzero then $(B, 0)$ is a Banach SN space without a Banach SN dual. We will have a significant example of a Banach SN space with a Banach SN dual in Example 6.1.

We suppose from now on that $(B, L)$ is a Banach SN space with a Banach SN dual $\left(B^{*}, \widetilde{L}\right)$. We note then from $(5)$ that,

$$
b \in B \quad \Longrightarrow \quad q_{\widetilde{L}}(L b)=\frac{1}{2}\langle L b, \widetilde{L} L b\rangle=\frac{1}{2}\langle b, L b\rangle=q_{L}(b) .
$$

We will use the following result in Corollary 5.12.

Lemma 4.2. Let $L(B)=B^{*}$ and $A$ be a maximally $L$-positive subset of $B$. Then

$$
c^{*} \in B^{*} \Longrightarrow \inf _{a \in A} q_{\widetilde{L}}\left(L a-c^{*}\right) \leq 0 .
$$

Proof. Let $c^{*} \in B^{*}$, and $b \in B$ be chosen so that $L b=c^{*}$. Then, from (6) and (4),

$$
\inf _{a \in A} q_{\widetilde{L}}\left(L a-c^{*}\right)=\inf _{a \in A} q_{\widetilde{L}}(L a-L b)=\inf _{a \in A} q_{L}(a-b) \leq 0 .
$$

In order to simplify notation in what follows, we will write $\Lambda:=-\widetilde{L}$, so $\left(B^{*}, \Lambda\right)$ is also a Banach SN space. We note then from (5) and (6) that

$$
b \in B \text { and } b^{*} \in B^{*} \quad \Longrightarrow \quad\left\langle b, b^{*}\right\rangle=-\left\langle L b, \Lambda b^{*}\right\rangle \text {, }
$$

and

$$
b \in B \quad \Longrightarrow \quad-q_{L}(b)=q_{\Lambda}(L b) .
$$

\section{Closed linear $L$-positive subspaces}

We will use the following standard notation and results from convex analysis. If $f: B \rightarrow$ ]$-\infty, \infty]$ is proper and convex and $b \in B$ then the subdifferential of $f$ at $b, \partial f(b)$, is the subset of $B^{*}$ defined by

$$
b^{*} \in \partial f(b) \Longleftrightarrow \text { for all } c \in B, f(b)+\left\langle c-b, b^{*}\right\rangle \leq f(c) .
$$

We now give a result on the existence of "almost horizontal" subtangents: 


\section{Polar subspaces and automatic maximality}

Lemma 5.1. Let $f: B \rightarrow]-\infty, \infty]$ be proper, convex, lower semicontinuous and bounded below, and $\eta>0$. Then there exist $b \in \operatorname{dom} f$ and $d^{*} \in \partial f(b)$ such that $\left\|d^{*}\right\| \leq \eta$.

Proof. This follows from the Brøndsted-Rockafellar Theorem (see Brøndsted-Rockafellar, [6, Lemma, pp. 608-609], Zălinescu, [16, Theorem 3.1.2, p. 161], or [12, Theorem 18.6, p. 76]).

For the rest of this section, we shall suppose that $A$ is a closed linear $L$-positive subspace of $B$ and $d \in B$. We define the functions $\left.\left.q_{L}^{A, d}: B \rightarrow\right]-\infty, \infty\right]$ and $q_{L}^{A}: B \rightarrow$ ]$-\infty, \infty]$ by

$$
q_{L}^{A, d}(b):=\left\{\begin{array}{ll}
q_{L}(b) & (\text { if } b \in A-d) ; \\
\infty & \text { (otherwise) }
\end{array} \quad \text { and } \quad q_{L}^{A}(b):=q_{L}^{A, 0}(b)= \begin{cases}q_{L}(b) & \text { (if } b \in A) \\
\infty & \text { (otherwise) }\end{cases}\right.
$$

Lemma 5.2 $q_{L}^{A, d}$ is proper and convex and

$$
\partial q_{L}^{A, d}(b)= \begin{cases}L b+A^{0} & (\text { if } b \in A-d) \\ \emptyset & \text { (otherwise) }\end{cases}
$$

Proof. See [14, Lemmas 5.1 and 5.2, p. 609].

The following result is well known:

Lemma 5.3 Let $\eta>0, g: B \rightarrow \mathbb{R}$ be defined by $g:=2 \eta\|\cdot\|, b \in B$ and $b^{*} \in B^{*}$. Then $b^{*} \in \partial g(b) \Longleftrightarrow\left\|b^{*}\right\| \leq 2 \eta$ and $\left\langle b, b^{*}\right\rangle=2 \eta\|b\|$.

Remark 5.4. The technical result that follows is the central result of this paper. At this time, we point out that the main difference between it and its antecedent, [14, Theorem $5.3(\mathrm{a})$, pp. 609-610], is that we use a penalty function of the form $2 \eta\|\cdot\|$ rather than $\frac{1}{2}\|\cdot\|^{2}$. We will explain the implications of this in Remark 5.8.

Lemma 5.5. Let $A$ be a closed linear $L$-positive subspace of $B, A^{0}$ be $\Lambda$-positive, $d \in B, c^{*} \in B^{*}, \delta:=\inf _{a \in A}\|a-d\|$ and $\eta>0$. Then there exists $b \in A-d$ such that $\eta \delta \leq q_{\Lambda}\left(L b-c^{*}\right)+3\left\|c^{*}\right\| \eta+5 \eta^{2}$.

Proof. Let $g$ be as in Lemma 5.3, and write $f:=q_{L}^{A, d}+g-c^{*}$. If $\inf _{B} f=-\infty$ then we choose $b \in \operatorname{dom} f=A-d$ such that $f(b) \leq q_{\Lambda}\left(c^{*}\right)+3\left\|c^{*}\right\| \eta+5 \eta^{2}$. From (8) and (7),

$$
\begin{aligned}
\eta \delta & \leq 2 \eta \delta \leq g(b)=f(b)-q_{L}^{A, d}(b)+\left\langle b, c^{*}\right\rangle=f(b)-q_{L}(b)+\left\langle b, c^{*}\right\rangle \\
& =f(b)+q_{\Lambda}(L b)-\left\langle L b, \Lambda c^{*}\right\rangle=f(b)+q_{\Lambda}\left(L b-c^{*}\right)-q_{\Lambda}\left(c^{*}\right) \\
& \leq q_{\Lambda}\left(L b-c^{*}\right)+3\left\|c^{*}\right\| \eta+5 \eta^{2}
\end{aligned}
$$

which gives the required result. Thus we can and will suppose that $\inf _{B} f>-\infty$. From $(3), q_{L}$ is continuous on $B$. Since $A$ is closed in $B, f$ is lower semicontinuous on $B$. From Lemma 5.1, there exist $b \in \operatorname{dom} f=A-d$ and $d^{*} \in \partial f(b)$ such that $\left\|d^{*}\right\| \leq$ $\eta$. Since $g$ and $c^{*}$ are continuous, Rockafellar's formula for the subdifferential of a sum 


\section{Polar subspaces and automatic maximality}

(see Rockafellar, [10, Theorem 3(b), p. 85], Zălinescu, [16, Theorem 2.8.7(iii), p. 127], or [12, Theorem 18.1, pp. 74-75]) provide

$$
a^{*} \in \partial q_{L}^{A, d}(b) \text { and } b^{*} \in \partial g(b) \text { such that } d^{*}=a^{*}+b^{*}-c^{*} \text {, thus } a^{*}=c^{*}+d^{*}-b^{*} \text {. }
$$

Lemmas 5.2 and 5.3 imply that

$$
a^{*} \in L b+A^{0},\left\|b^{*}\right\| \leq 2 \eta, \text { and }\left\langle b, b^{*}\right\rangle=2 \eta\|b\| \text {. }
$$

Using (9), (10) and the facts that $\left\langle b, d^{*}\right\rangle \leq\|b\|\left\|d^{*}\right\| \leq \eta\|b\|$ and $\delta \leq\|b\|$,

$$
\eta \delta+\left\langle b, a^{*}\right\rangle=\eta \delta+\left\langle b, c^{*}\right\rangle+\left\langle b, d^{*}\right\rangle-\left\langle b, b^{*}\right\rangle \leq \eta \delta+\left\langle b, c^{*}\right\rangle+\eta\|b\|-2 \eta\|b\| \leq\left\langle b, c^{*}\right\rangle .
$$

Thus, combining with (7),

$$
\eta \delta-\left\langle L b, \Lambda a^{*}\right\rangle \leq-\left\langle L b, \Lambda c^{*}\right\rangle
$$

From (9) again, $\left\|a^{*}-c^{*}\right\| \leq\left\|d^{*}\right\|+\left\|b^{*}\right\| \leq \eta+2 \eta=3 \eta$ thus, applying (2) to $\left(B^{*}, q_{\Lambda}\right)$,

$$
\left|q_{\Lambda}\left(a^{*}\right)-q_{\Lambda}\left(c^{*}\right)\right| \leq \frac{1}{2}\left\|a^{*}-c^{*}\right\|\left\|a^{*}+c^{*}\right\| \leq \frac{1}{2} 3 \eta\left(3 \eta+\left\|2 c^{*}\right\|\right) \leq 3\left\|c^{*}\right\| \eta+5 \eta^{2} .
$$

From the $\Lambda$-positivity of $A^{0},(10),(11)$ and (12),

$$
\begin{aligned}
\eta \delta & \leq \eta \delta+q_{\Lambda}\left(L b-a^{*}\right)=\eta \delta+q_{\Lambda}(L b)-\left\langle L b, \Lambda a^{*}\right\rangle+q_{\Lambda}\left(a^{*}\right) \\
& \leq q_{\Lambda}(L b)-\left\langle L b, \Lambda c^{*}\right\rangle+q_{\Lambda}\left(c^{*}\right)+3\left\|c^{*}\right\| \eta+5 \eta^{2}=q_{\Lambda}\left(L b-c^{*}\right)+3\left\|c^{*}\right\| \eta+5 \eta^{2} .
\end{aligned}
$$

This completes the proof of Lemma 5.5.

In fact, we will not need Lemma 5.5 in full generality. We will need the following two special cases: Corollary 5.6 is obtained by setting $c^{*}=0$ and noting from (8) that $q_{\Lambda}(L b)=-q_{L}(b)$, and Corollary 5.7 is obtained by setting $d=0$ and noting that $\eta \delta \geq 0$.

Corollary 5.6. Let $A$ be a closed linear $L$-positive subspace of $B, A^{0}$ be $\Lambda$-positive, $d \in B$ and $\eta>0$. Then there exists $b \in A-d$ such that $\eta \inf _{a \in A}\|a-d\|+q_{L}(b) \leq 5 \eta^{2}$.

Corollary 5.7. Let $A$ be a closed linear $L$-positive subspace of $B, A^{0}$ be $\Lambda$-positive, $c^{*} \in B^{*}$ and $\eta>0$. Then there exists $b \in A$ such that $0 \leq q_{\Lambda}\left(L b-c^{*}\right)+3\left\|c^{*}\right\| \eta+5 \eta^{2}$.

Remark 5.8. With no extra effort, one can strengthen the conclusion of Lemma 5.5 to there exists $b \in A-d$ such that $\eta\|b\| \leq q_{\Lambda}\left(L b-c^{*}\right)+3\left\|c^{*}\right\| \eta+5 \eta^{2}$. Theorem 5.9(a) below was proved in [14, Theorem 5.3(d), pp. 609-611]. Theorem 5.9(b) depends on Lemma 3.3, of which we were not aware when [14] was written. Theorem 5.9(c) is a considerable generalization of the critical part of [14, Corollary 5.4, p. 611], which assumed the additional condition that, for all $d^{*} \in B^{*}, \inf _{d \in B}\left[q_{\widetilde{L}}\left(d^{*}-L d\right)+\frac{1}{2}\left\|d^{*}-L d\right\|^{2}\right] \leq 0$. We do not know exactly why the change of penalty function mentioned in Remark 5.4 seems to produce this stronger result. (Of course, $\Lambda$-positivity is equivalent to $\widetilde{L}$-negativity.) 


\section{Polar subspaces and automatic maximality}

Theorem 5.9. Let $A$ be a closed linear $L$-positive subspace of $B$ and $A^{0}$ be $\Lambda$-positive. Then:

(a) $A$ is maximally $L$-positive.

(b) $A^{0}$ is maximally $\Lambda$-positive.

(c) For all $c^{*} \in B^{*}, \inf _{a \in A} q_{\widetilde{L}}\left(L a-c^{*}\right) \leq 0$.

Proof. (a) Let $d \in B$ and $\{d\} \cup A$ be $L$-positive. Corollary 5.6 provides us with $b \in A-d$ such that $\eta \inf _{a \in A}\|a-d\|+q_{L}(b) \leq 5 \eta^{2}$. By hypothesis, $q_{L}(b) \geq 0$, hence $\eta \inf _{a \in A}\|a-d\| \leq$ $5 \eta^{2}$. Thus $\inf _{a \in A}\|a-d\| \leq 5 \eta$. Since this holds for all $\eta>0$ and $A$ is closed, $d \in A$. This completes the proof that $A$ is maximally $L$-positive.

(b) Let $c^{*} \in B$ and $\left\{c^{*}\right\} \cup A^{0}$ be $\Lambda$-positive. Write $Z$ for the linear subspace $\left\{b \in B:\left\langle b, c^{*}\right\rangle=0\right\}$ of $B . A \cap Z$ is obviously a closed linear subspace of $B$ and, since $A \cap Z \subset A, A \cap Z$ is $L$-positive. However, from Lemma 2.2, $(A \cap Z)^{0}=\operatorname{lin}\left\{c^{*}, A^{0}\right\}$ and, from Lemma 3.3 (applied to the $\mathrm{SN}$ space $\left(B^{*}, \Lambda\right)$ ), $\operatorname{lin}\left\{c^{*}, A^{0}\right\}$ is $\Lambda$-positive. Thus, applying (a) with $A$ replaced by $A \cap Z, A \cap Z$ is maximally $L$-positive. Since $A$ is $L$-positive and $A \cap Z \subset A$, in fact $A \cap Z=A$, that is to say $A \subset Z$. Consequently $\left\langle A, c^{*}\right\rangle=\{0\}$, that is to say $c^{*} \in A^{0}$. This completes the proof that $A^{0}$ is maximally $\Lambda$-positive.

(c) Let $c^{*} \in B^{*}$. Corollary 5.7 provides us with $b \in A$ such that $0 \leq q_{\Lambda}\left(L b-c^{*}\right)+$ $3\left\|c^{*}\right\| \eta+5 \eta^{2}$. Equivalently, $q_{\widetilde{L}}\left(L b-c^{*}\right) \leq 3\left\|c^{*}\right\| \eta+5 \eta^{2}$. The result now follows by letting $\eta \rightarrow 0$.

Remark 5.10. Our next result is an improved version of [14, Corollary 5.4, p. 611]. The critical parts are the implications $(13) \Longrightarrow(14)$ and $(13) \Longrightarrow(16)$, which are true without any additional conditions. As pointed out in Remark 5.8, in [14], we assumed the additional condition that, for all $d^{*} \in B^{*}, \inf _{d \in B}\left[q_{\widetilde{L}}\left(d^{*}-L d\right)+\frac{1}{2}\left\|d^{*}-L d\right\|^{2}\right] \leq 0$ for the first implication, and we did not establish the second implication.

Corollary 5.11. Let $A$ be a closed linear $L$-positive subspace of $B$. Then the conditions (13)-(16) are equivalent.

$$
A^{0} \text { is } \Lambda \text {-positive. }
$$

$A$ is maximally $L-$ positive and, for all $c^{*} \in B^{*}, \inf _{a \in A} q_{\widetilde{L}}\left(L a-c^{*}\right) \leq 0$.

$$
\begin{gathered}
\text { For all } c^{*} \in B^{*}, \inf _{a \in A} q_{\widetilde{L}}\left(L a-c^{*}\right) \leq 0 . \\
A^{0} \text { is maximally } \Lambda \text {-positive. }
\end{gathered}
$$

Proof. It is clear from Theorem 5.9 (a,c) that $(13) \Longrightarrow(14)$, and it is obvious that $(14) \Longrightarrow$ (15). If (15) is true then, for all $c^{*} \in A^{0}$,

$$
\inf _{a \in A} q_{L}(a)+q_{\widetilde{L}}\left(c^{*}\right)=\inf _{a \in A}\left[q_{L}(a)-\left\langle a, c^{*}\right\rangle+q_{\widetilde{L}}\left(c^{*}\right)\right]=\inf _{a \in A} q_{\widetilde{L}}\left(L a-c^{*}\right) \leq 0 .
$$

Since $\inf _{a \in A} q_{L}(a) \geq 0$, it follows that $q_{\widetilde{L}}\left(c^{*}\right) \leq 0$, from which $q_{\Lambda}\left(c^{*}\right) \geq 0$, and the linearity of $A^{0}$ gives (13). Thus (13)-(15) are equivalent. Clearly, (16) $\Longrightarrow(13)$, and the reverse implication is immediate from Theorem 5.9(b).

Corollary 5.12. Let $A$ be a closed linear $L$-positive subspace of $B$ and $L(B)=B^{*}$. Then $A$ is maximally $L$-positive if, and only if, $A^{0}$ is $\Lambda$-positive. 


\section{Polar subspaces and automatic maximality}

Proof. If $A^{0}$ is $\Lambda$-positive then Theorem 5.9(a) implies directly that $A$ is maximally $L-$ positive. If, conversely, $A$ is maximally $L$-positive then Lemma 4.2 implies that (15) is satisfied, and it then follows from Corollary 5.11 that $A^{0}$ is $\Lambda$-positive.

\section{Monotone sets, type (NI) and linear monotone subspaces}

We suppose in this section that $E$ is a nonzero Banach space.

Example 6.1. Let $B:=E \times E^{*}$ and, for all $\left(x, x^{*}\right) \in B,\left\|\left(x, x^{*}\right)\right\|:=\sqrt{\|x\|^{2}+\left\|x^{*}\right\|^{2}}$. We represent $B^{*}$ by $E^{*} \times E^{* *}$, under the pairing $\left\langle\left(x, x^{*}\right),\left(y^{*}, y^{* *}\right)\right\rangle:=\left\langle x, y^{*}\right\rangle+\left\langle x^{*}, y^{* *}\right\rangle$, and define $L: B \rightarrow B^{*}$ by $L\left(x, x^{*}\right):=\left(x^{*}, \widehat{x}\right)$. Then $(B, L)$ is a Banach $\mathrm{SN}$ space and, for all $\left(x, x^{*}\right) \in B, q_{L}\left(x, x^{*}\right)=\frac{1}{2}\left[\left\langle x, x^{*}\right\rangle+\left\langle x, x^{*}\right\rangle\right]=\left\langle x, x^{*}\right\rangle$. If $\left(x, x^{*}\right),\left(y, y^{*}\right) \in B$ then we have $q_{L}\left(\left(x, x^{*}\right)-\left(y, y^{*}\right)\right)=q_{L}\left(x-y, x^{*}-y^{*}\right)=\left\langle x-y, x^{*}-y^{*}\right\rangle$. Thus if $A \subset B$ then $A$ is $L$-positive exactly when $A$ is a nonempty monotone subset of $B$ in the usual sense, and $A$ is maximally $L$-positive exactly when $A$ is a maximally monotone subset of $B$ in the usual sense. We point out that any finite dimensional Banach SN space of the form described here must have even dimension, and that there are many Banach SN spaces of finite odd dimension with Banach SN duals. See [13, Remark 6.7, p. 246].

As usual, the dual norm on $B^{*}=E^{*} \times E^{* *}$ is given by $\left\|\left(y^{*}, y^{* *}\right)\right\|:=\sqrt{\left\|y^{*}\right\|^{2}+\left\|y^{* *}\right\|^{2}}$. By analogy with the analysis above, we define $\widetilde{L}: B^{*} \rightarrow B^{* *}$ by $\widetilde{L}\left(y^{*}, y^{* *}\right)=\left(y^{* *}, \widehat{y^{*}}\right)$. Then $\left(B^{*}, \widetilde{L}\right)$ is a Banach SN space, and, for all $\left(y^{*}, y^{* *}\right) \in B^{*}, q_{\widetilde{L}}\left(y^{*}, y^{* *}\right)=\left\langle y^{*}, y^{* *}\right\rangle$. Now let $b=\left(x, x^{*}\right) \in B$ and $b^{*}=\left(y^{*}, y^{* *}\right) \in B^{*}$. Then $\left\langle L b, \widetilde{L} b^{*}\right\rangle=\left\langle\left(x^{*}, \widehat{x}\right),\left(y^{* *}, \widehat{y}^{*}\right)\right\rangle=$ $\left\langle x^{*}, y^{* *}\right\rangle+\left\langle\widehat{x}, \widehat{y}^{*}\right\rangle=\left\langle x^{*}, y^{* *}\right\rangle+\left\langle x, y^{*}\right\rangle=\left\langle x, y^{*}\right\rangle+\left\langle x^{*}, y^{* *}\right\rangle=\left\langle\left(x, x^{*}\right),\left(y^{*}, y^{* *}\right)\right\rangle=\left\langle b, b^{*}\right\rangle$. Thus (5) is satisfied, and so $\left(B^{*}, \widetilde{L}\right)$ is a Banach $\mathrm{SN}$ dual of $(B, L)$. As in the general case, we define $\Lambda\left(y^{*}, y^{* *}\right):=-\widetilde{L}\left(y^{*}, y^{* *}\right)=-\left(y^{* *}, \widehat{y^{*}}\right)$.

Definition 6.2 first appeared in [11, Definition 10, p. 183]. It was thought at first that this was a weak definition, but it was proved by Marques Alves and Svaiter in [9, Theorem 4.4, pp. 1084-1085], that if $A$ is a maximally monotone subset of $E \times E^{*}$ of type (NI) then $A$ is of "type $(D)$ " (the opposite implication is obviously true). This result was extended in [13, Theorem 9.9 (a), pp. 254-255], where it was proved that if $A$ is a maximally monotone subset of $E \times E^{*}$ of type (NI) then $A$ is of "dense type" and "type (ED)". This has a number of consequences, which are detailed in [13, Theorem 9.9(b-f), pp. 254-255] and [13, Theorem 9.10, pp. 255-256]. Finally, it was proved by Bauschke, Borwein, Wang and Yao in [4, Theorem 3.1, pp. 1878-1879] that if $A$ is maximally monotone subset of $E \times E^{*}$ then $A$ is of type (NI) if, and only if, $A$ is of "Fitzpatrick-Phelps" type.

What distinguishes the definition of "type (NI)" from the definitions of the other classes of maximally monotone sets mentioned above is that it can be recast easily in the language of Banach SN spaces, as we will see in Lemma 6.3 below.

Definition 6.2. Let $A \subset E \times E^{*}$. We say that $A$ is of type (NI) if,

$$
\text { for all }\left(y^{*}, y^{* *}\right) \in E^{*} \times E^{* *}, \quad \inf _{\left(x, x^{*}\right) \in A}\left\langle x^{*}-y^{*}, \widehat{x}-y^{* *}\right\rangle \leq 0 .
$$

Here is the promised reformulation of Definition 6.2 in the notation of Banach SN spaces. We use the conventions introduced in Example 6.1. 


\section{Polar subspaces and automatic maximality}

Lemma 6.3 Let $A \subset B:=E \times E^{*}$. Then $A$ is of type (NI) if, and only if, for all $c^{*} \in B^{*}$, $\inf _{a \in A} q \widetilde{L}\left(L a-c^{*}\right) \leq 0$.

Proof. This is immediate from the formulae for $q_{\widetilde{L}}$ and $L$ given in Example 6.1.

Let $A$ be a linear subspace of $E \times E^{*}$. The adjoint subspace, $A^{\mathrm{T}}$, of $E^{* *} \times E^{*}$, is defined by: $\quad\left(y^{* *}, y^{*}\right) \in A^{\mathrm{T}} \Longleftrightarrow$ for all $\left(a, a^{*}\right) \in A,\left\langle a, y^{*}\right\rangle=\left\langle a^{*}, y^{* *}\right\rangle$. This definition goes back at least to Arens in [1]. (We use the notation " $A^{\mathrm{T}}$ " rather than the more usual " $A^{*}$ " to avoid confusion with the dual space of $A$.) It is clear that

$$
\left(y^{* *}, y^{*}\right) \in A^{\mathrm{T}} \Longleftrightarrow\left(y^{*},-y^{* *}\right) \in A^{0} .
$$

Now, for all $\left(y^{*}, y^{* *}\right) \in B^{*}=E^{*} \times E^{* *}, q_{\Lambda}\left(y^{*}, y^{* *}\right)=-\left\langle y^{*}, y^{* *}\right\rangle$, from which it follows easily by tracking through the changes of sign and the order of the variables that $A^{\mathrm{T}}$ is a monotone subset of $E^{* *} \times E^{*}$ if, and only if, $A^{0}$ is a $\Lambda$-positive subset of $B^{*}$, and $A^{\mathrm{T}}$ is a maximally monotone subset of $E^{* *} \times E^{*}$ if, and only if, $A^{0}$ is a maximally $\Lambda$-positive subset of $B^{*}$.

We will now see how the results of Section 5 translate into our present context. Our first result, which we have not seen in the literature, is about "automatic maximality":

Theorem 6.4. Let $A$ be a closed linear monotone subspace of $E \times E^{*}$ and $A^{\mathrm{T}}$ be a monotone subspace of $E^{* *} \times E^{*}$. Then $A$ and $A^{\mathrm{T}}$ are maximally monotone in their respective spaces.

Proof. This is immediate from Theorem 5.9(a,b).

Remark 6.5. The implication $(17) \Longrightarrow(18)$ below was shown by Bauschke, Borwein, Wang and Yao in a two-stage process as follows: It was first shown in [3, Theorem 3.1 (iii) $\Longrightarrow$ (ii), pp. 6-9] that if $A$ is a maximally monotone linear subspace of $E \times E^{*}$ and (17) is satisfied then $A$ is of type (NI), and it was subsequently shown in [5, Propositions 3.1-2, pp. 49554959] that if $A$ is a closed monotone linear subspace of $E \times E^{*}$ and (17) is satisfied then $A$ is maximally monotone. Both of these proofs are algebraically quite complicated, and depend ultimately on the penalty function $\left(x, x^{*}\right) \mapsto \frac{1}{2}\|x\|^{2}+\frac{1}{2}\left\|x^{*}\right\|^{2}$. See Remark 5.4.

As far as we know, the implication $(17) \Longrightarrow(19)$ in this generality has not been observed before. It was proved in the reflexive case in Yao, [15, Theorems 18.4-5, p. 394-395]. It was proved for general Banach spaces and "skew" subspaces by Bauschke, Borwein, Wang and Yao in [5, Corollary 4.4, p. 4961] and for subspaces whose domain (i.e., the projection onto $E)$ is also closed in [5, Theorem $5.5((\mathrm{v}) \Longleftrightarrow(\mathrm{vi}))$, pp. 4965-4968].

Corollary 6.6. Let $A$ be a closed linear monotone subspace of $E \times E^{*}$. Then the four conditions below are equivalent.

$$
\begin{gathered}
A^{\mathrm{T}} \text { is a monotone subspace of } E^{* *} \times E^{*} . \\
A \text { is maximally monotone of type }(\mathrm{NI}) . \\
A \text { is of type }(\mathrm{NI}) . \\
A^{\mathrm{T}} \text { is a maximally monotone subspace of } E^{* *} \times E^{*} .
\end{gathered}
$$

Proof. This is immediate from Corollary 5.11 and Lemma 6.3. 


\section{Polar subspaces and automatic maximality}

Corollary 6.7 below appears in Brezis-Browder [2, Theorems 1-2, pp. 32-39]. (The result actually proved in [2, Theorem 1] is stronger than that which appears in Corollary 6.7.)

Corollary 6.7. Let $E$ be reflexive and $A$ be a closed linear monotone subspace of $E \times E^{*}$. Then $A$ is maximally monotone if, and only if, $A^{\mathrm{T}}$ is monotone.

Proof. This follows from Corollary 5.12 and the comments in Example 6.1.

\section{References}

[1] R. Arens, Operational calculus of linear relations, Pacific J. Math. 11 (1961) 9-23.

[2] H. Brezis and F. E. Browder, Linear maximal monotone operators and singular nonlinear integral equations of Hammerstein, type, Nonlinear analysis (collection of papers in honor of Erich H. Rothe), pp. 31-42. Academic Press, New York, 1978.

[3] H. Bauschke, J. M. Borwein, X. Wang and L. Yao For maximally monotone linear relations, dense type, negative-infimum type, and Fitzpatrick-Phelps type all coincide with monotonicity of the adjoint, http://arxiv.org/abs/1103.6239v1, posted March 31, 2011.

[4] H. Bauschke, J. M. Borwein, X. Wang and L. Yao Every maximally monotone operator of Fitzpatrick-Phelps type is actually of dense type, Optim Lett 6 (2012), 1875-1881. DOI 10.1007/s11590-011-0383-2.

[5] H. Bauschke, J. M. Borwein, X. Wang and L. Yao The Brezis-Browder Theorem in a general Banach space, Journal of Functional Analysis 262(2012), 4948-4971. http://dx.doi.org/10.1016/j.jfa.2012.03.023.

[6] A. Brøndsted and R.T. Rockafellar, On the Subdifferentiability of Convex Functions, Proc. Amer. Math. Soc. 16(1965), 605-611.

[7] Y. García Ramos, J. E. Martínez-Legaz and S. Simons, New results on q-positivity, Positivity 16(2012), 543-563. DOI 10.1007/s11117-012-0191-7

[8] J. L. Kelley, I. Namioka, and co-authors, Linear Topological Spaces, D. Van Nostrand Co., Inc., Princeton - Toronto - London - Melbourne (1963).

[9] M. Marques Alves and B. F. Svaiter, On Gossez type (D) maximal monotone operators., J. of Convex Anal., 17(2010), 1077-1088.

[10] R. T. Rockafellar, Extension of Fenchel's duality theorem for convex functions, Duke Math. J. 33(1966), 81-89.

[11] S. Simons, The range of a monotone operator, J. Math. Anal. Appl. 199(1996), 176-201.

[12] —- From Hahn-Banach to monotonicity, Lecture Notes in Mathematics, 1693, second edition, (2008), Springer-Verlag.

[13] — - Banach SSD spaces and classes of monotone sets, J. of Convex Anal. 18(2011), $227-258$.

[14] — Linear L-positive sets and their polar subspaces, Set-Valued and Variational Anal. 20(2012), 603-615. DOI: 10.1007/s11228-012-0206-3.

[15] L. Yao, The Brezis-Browder theorem revisited and properties of Fitzpatrick functions of order $n$, Fixed-point algorithms for inverse problems in science and engineering, Springer Optim. Appl., 49(2011), 391-402. 
Polar subspaces and automatic maximality

[16] C. Zălinescu, Convex analysis in general vector spaces, (2002), World Scientific. 